\title{
SalsaNet: Fast Road and Vehicle Segmentation in LiDAR Point Clouds for Autonomous Driving
}

\author{
Eren Erdal Aksoy ${ }^{1,2}$, Saimir $\mathrm{Baci}^{2}$, and Selcuk Cavdar ${ }^{2}$
}

\begin{abstract}
In this paper, we introduce a deep encoderdecoder network, named SalsaNet, for efficient semantic segmentation of 3D LiDAR point clouds. SalsaNet segments the road, i.e. drivable free-space, and vehicles in the scene by employing the Bird-Eye-View (BEV) image projection of the point cloud. To overcome the lack of annotated point cloud data, in particular for the road segments, we introduce an autolabeling process which transfers automatically generated labels from the camera to LiDAR. We also explore the role of imagelike projection of LiDAR data in semantic segmentation by comparing BEV with spherical-front-view projection and show that SalsaNet is projection-agnostic. We perform quantitative and qualitative evaluations on the KITTI dataset, which demonstrate that the proposed SalsaNet outperforms other state-ofthe-art semantic segmentation networks in terms of accuracy and computation time. Our code and data are publicly available at https://gitlab.com/aksoyeren/salsanet.git
\end{abstract}

\section{INTRODUCTION}

Semantic segmentation of 3D point cloud data plays a key role in scene understanding to reach full autonomy in self-driving vehicles. For instance, estimating the free drivable space together with vehicles in the front can lead to safe maneuver planning and decision making, which enables autonomous driving to a great extent.

Recently, great progress has been made in deep learning to generate accurate, real-time and robust semantic segments. Most of these advanced segmentation approaches heavily rely on camera data [1], [2], [3]. In contrast to passive camera sensors, 3D LiDARs (Light Detection And Ranging) have wider field of view and provide significantly reliable distance measurement robust to environmental illumination. Thus, 3D LiDAR scanners have always been an important component in the perception pipeline of autonomous vehicles.

Unlike images, LiDAR point clouds are, however, relatively sparse and contain a vast number of irregular, i.e. unstructured, points. In addition, the density of points varies drastically due to non-uniform sampling of the environment, which makes the intensive point searching and indexing operations relatively expensive. Among others, a common attempt to tackle all these challenges is to project point clouds into a $2 \mathrm{D}$ image space in order to generate a structured (matrix) form required for the standard convolution process. Existing 2D projections are Bird-Eye-View (BEV) (i.e. top view) and Spherical-Front-View (SFV) (i.e. panoramic view).

\footnotetext{
The research leading to these results has received funding from the Vinnova FFI project SHARPEN, under grant agreement no. 2018-05001.

${ }^{1}$ Halmstad University, School of Information Technology, Center for Applied Intelligent Systems Research, Halmstad, Sweden

${ }^{2}$ Volvo Technology AB, Volvo Group Trucks Technology, Vehicle Automation, Gothenburg, Sweden
}

However, to the best of our knowledge, there is still no comprehensive comparative study showing the contribution of these projection methods in the segmentation process.

In the context of semantic segmentation of 3D LiDAR data, most of the recent studies employ these projection methods to focus on the estimation of either the road itself [4], [5] or only the obstacles on the road (e.g. vehicles) [6], [7]. All these segments are, however, equally important for the subsequent navigation components (e.g. maneuver planning) and, thus, need to be jointly processed. The main reason of having this decoupled treatment in the literature is the lack of large annotated point cloud data, in particular, for the road segments.

In this paper, we study the joint segmentation of the road, i.e. drivable free-space, and vehicles using 3D LiDAR point clouds only. We propose a novel "SemAntic Lidar data SegmentAtion Network", i.e. SalsaNet, which has an encoder-decoder architecture where the encoder part contains consecutive ResNet blocks [8]. Decoder part rather upsamples features and combines them with the corresponding counterparts from the early residual blocks via skip connections. Final output of the decoder is then sent to the pixelwise classification layer to return semantic segments.

We validate our network's performance on the KITTI dataset [9] which provides 3D bounding boxes for vehicles and a relatively small number of annotated road images $(\approx 300$ samples). Inspired from [10], [11], we propose an auto-labeling process to automatically label $\approx 11 \mathrm{~K}$ point clouds in the KITTI dataset. For this purpose, we employ the state-of-the-art methods [12] and [13] to respectively segment road and vehicles in camera images. These segments are then mapped from camera space to LiDAR to automatically generate annotated point clouds.

The input of SalsaNet is the BEV rasterized image format of the point cloud where each image channel stores a unique statistical cue (e.g. average depth and intensity values). To further analyze the role of the point cloud projection in the network performance, we separately train SalsaNet with the SFV representation and provide a comprehensive comparison with the BEV counterpart.

Quantitative and qualitative experiments on the KITTI dataset show that the proposed SalsaNet is projectionagnostic, i.e. exhibiting high performance in both projection methods and significantly outperforms other state-of-the-art semantic segmentation approaches [6], [7], [14] in terms of pixel-wise segmentation accuracy while requiring much less computation time.

Our contribution is manifold: 
- We introduce an encoder-decoder architecture to semantically segment road and vehicle points in real-time using 3D LiDAR data only.

- To automatically annotate a large set of 3D LiDAR point clouds, we transfer labels across different sensor modalities, e.g. from camera images to LiDAR.

- We study two commonly used point cloud projection methods and compare their effects in the semantic segmentation process in terms of accuracy and speed.

- We provide a thorough quantitative comparison of the proposed SalsaNet on the KITTI dataset with different state-of-the-art semantic segmentation networks.

- We also release our source code and annotated point clouds to encourage further research.

\section{RELATED WORK}

There exist multiple prior methods exploring the semantic segmentation of 3D LiDAR point cloud data. Those methods are basically classified under two categories. The first one involves conventional heuristic approaches such as model fitting by employing iterative approaches [15] or histogram computation after projecting LiDAR point clouds to $2 \mathrm{D}$ space [16]. In contrast, the second category investigates advanced deep learning approaches [6], [7], [17], [18] which achieved a quantum jump in performance during the last decade. These approaches in the latter class differ from each other not only in terms of network architecture but also in the way the LiDAR data is represented before being fed to the network.

Regarding the network architecture, high performance segmentation methods particularly use fully convolutional networks [19], encoder-decoder structures [20], or multi-branch models [1]. The main difference between these architectures is the way of encoding features at various depths and later incorporating them for recovering the spatial information. We, in this study, follow the encoder-decoder structure due to the high performance observed in the recent state-of-theart point cloud segmentation studies [6], [7], [14].

In the context of $3 \mathrm{D}$ LiDAR point cloud representation, there exist three mainstream methods: voxel creation [21], [20], point-wise operation [22], and image projection [6], [7], [17]. Voxel representation transforms a point cloud into a high-dimensional volumetric form, i.e. 3D voxel grid [21], [20]. Due to sparsity in point clouds, the voxel grid, however, may have empty voxels which leads to redundant computations. Point-wise methods [22] process points directly without converting them into any other form. The main drawback here is the processing capacity which cannot efficiently handle large LiDAR point sets unless fusing them with additional cues from other sensory data, such as camera images as shown in [3]. To handle the sparsity in LiDAR point clouds, various image space projections, such as Bird-Eye-View (BEV) (i.e. top view) [4], [23], [24] and Spherical-Front-View (SFV) (i.e. panoramic view) [6], [7], [17], have been introduced. In contrast to voxel and point-wise approaches, the $2 \mathrm{D}$ projection is more compact, dense and, thus, amenable to real-time computation. In this study, our network relies on BEV since the SFV projection introduces distortion and uncommon deformation which has significant effects on relatively small objects such as vehicles.

Closest to our work are the recent studies in [6], [7]. Those approaches, however, segment only the road objects such as vehicles, pedestrians and cyclists using the SFV projection. In contrast, we only focus on road and vehicle segments in the poincloud. We exclude pedestrians and cyclists since their total number of instances in the entire KITTI dataset is significantly low which naturally yields poor learning performance as already shown in [6], [7].

There are also numerous networks addressing the road segmentation [4], [5]. These approaches, however, omit road objects (e.g. vehicles) and rely on limited annotated data ( $\approx 300$ samples) in the KITTI road dataset. To generate more training data, we automatically label road and vehicle segments in the entire KITTI point clouds $(\approx 11 \mathrm{~K}$ samples $)$ by transferring the camera image annotations derived by state-of-the-art segmentation methods [12], [13]. Note that a similar auto-labeling process has already been proposed in [10], [11] and a new large labeled 3D road scene point cloud dataset has been introduced in [20], however, the final labeled data has still not been released in any of these works for public use, which is not the case for our work.

\section{METHOD}

In this section, we give a detailed description of our proposed method starting with the automatic labeling of $3 \mathrm{D}$ point cloud data. We then continue with the point cloud representation, network architecture and training details.

\section{A. Data Labeling}

Lack of large annotated point cloud data introduces a challenge in the network training and evaluation. Applying crowdsourced manual data labeling is cumbersome due to a huge number of points in each individual scene cloud. Inspired from [10], [11], we, instead, propose an autolabeling process illustrated in Fig. 1 to automatically label 3D LiDAR point clouds.

Since the image-based detection and segmentation methods are relatively more mature than LiDAR-based solutions, we benefit from this stream of work to annotate 3D LiDAR point clouds. In this respect, to extract the road pixels, we use an off-the-shelf neural network MultiNet [12] dedicated to the road segmentation in camera images. We here note that the reason of using MultiNet is beacuse it is opensource and is already trained on the KITTI road detection benchmark [9]. To extract the vehicle points in the cloud, we employ Mask R-CNN [13] to segment labels in the camera image. Note that in case of having bounding box object annotations, as in the KITTI object detection benchmark [9], we directly label those points inside the $3 \mathrm{D}$ bounding box as vehicle segments. Finally, with the aid of the camera-LiDAR calibration, both road and vehicles segments are transferred from image space to the point cloud as shown in Fig. 1 


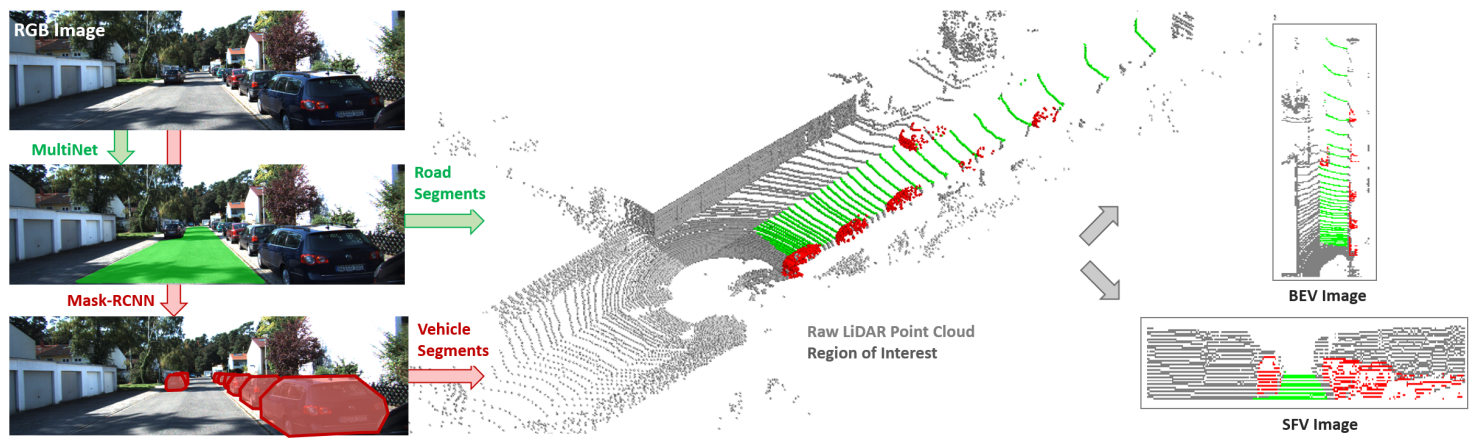

Fig. 1. Automatic point cloud labelling to generate network inputs in BEV and SFV formats.

\section{B. Point Cloud Representation}

Given an unstructured 3D LiDAR point cloud, we investigate the $2 \mathrm{D}$ grid-based image representation since it is more compact and yields real-time inference. As 3D points lies on a grid in the projected form, it also allows standard convolutions. We here note that reducing the dimension from 3 to 2 does not yield information loss in the point cloud since the height information is still kept as an additional channel in the projected image. Therefore, in this work, we exploit two common projections: Bird-Eye-View (BEV) and SphericalFront-View (SFV) representations.

1) Bird-Eye-View (BEV): We initially define a region-ofinterest in the raw point cloud, covering a large area in front of the vehicle which is $50 \mathrm{~m}$ long $(x \in[0,50])$ and $18 \mathrm{~m}$ wide $(y \in[-6,12])$ (see the dark gray points in Fig. 11. All the 3D points in this region-of-interest are then projected and discetized in to a 2D grid map with the size of $256 \times 64$. The grid map corresponds to the $x-y$ plane of the LiDAR and forms the BEV, i.e. top view projection of the point cloud. We set the grid cell sizes to 0.2 and 0.3 in $x-$ and $y$-axes, respectively. A sample BEV image is depicted in Fig. 1]

Similar to the work in [4], in each grid cell, we compute the mean and maximum elevation, average reflectivity (i.e. intensity) value, and number of projected points. Each of these four statistical information is encoded as one unique image channel, forming a 4D BEV image to be further used as the network input. Note that we also normalize each image channel to be within $[0,1]$. Compared to [4], we avoid using the minimum and standard deviation values of the height as additional features since our experiments showed that there is no significant contribution coming from those channels.

2) Spherical-Front-View (SFV): Following the work of [6], we also project the 3D point cloud onto a sphere to generate dense grid image representation in a rather panoramic view.

In this projection, each point is represented by two angles $(\theta, \phi)$ and an intensity $(i)$ value. In the $2 \mathrm{D}$ spherical grid image, each point is mapped to the coordinates $(u, v)$, where $u=\lfloor\theta / \Delta \theta\rfloor$ and $v=\lfloor\phi / \Delta \phi\rfloor$. Here, $\theta$ and $\phi$ are azimuth and zenith angles computed from point coordinates $(x, y, z)$ as $\theta=\arcsin \left(z / \sqrt{x^{2}+y^{2}+z^{2}}\right)$ and $\phi=\arcsin \left(y / \sqrt{x^{2}+y^{2}}\right)$, whereas $\Delta \theta$ and $\Delta \phi$ define the discretization resolutions.
For the projection, we mark the front-view area of $90^{\circ}$ as a region-of-interest. In each grid cell, we separately store 3D Cartesian coordinates $(x, y, z)$, the intensity value $(i)$ and range $r=\sqrt{x^{2}+y^{2}+z^{2}}$. As in [7], we also keep a binary mask indicating the occupancy of the grid cell. These six channels form the final image which has the resolution of $64 \times 512$. A sample SFV image is depicted in Fig. 1

Although SFV returns more dense representation compared to BEV, SFV has certain distortion and deformation effects on small objects, e.g. vehicles. It is also more likely that objects in SFV tend to occlude each other. We, therefore, employ BEV representation as the main input to our network. We, however, compare these two projections in terms of their contribution to the segmentation accuracy.

\section{Network Architecture}

The architecture of the proposed SalsaNet is depicted in Fig. 2. The input to SalsaNet is a $256 \times 64 \times 4 \mathrm{BEV}$ projection of the point cloud as described in Sec III-B.1.

SalsaNet has an encoder-decoder structure where the encoder part contains a series of ResNet blocks [8] (Block I in Fig. 2). Each ResNet block, except the very last one, is followed by dropout and pooling layers (Block II in Fig. 2). We employ max-pooling with kernel size of 2 to downsample feature maps in both width and height. Thus, on the encoder side, the total downsampling factor goes up to 16. Each convolutional layer has kernel size of 3 , unless otherwise stated. The number of feature channels are respectively $32,64,128,256$, and 256. Decoder network has a sequence of deconvolution layers, i.e. transposed convolutions (Blocks III in Fig. 2), to upsample feature maps, each of which is then element-wise added to the corresponding lower-level (bottom-up) feature maps of the same size transferred via skip connections (Blocks IV in Fig. 2). After each feature addition in the decoder, a stack of convolutional layers (Blocks V in Fig. 2) are introduced to capture more precise spatial cues to be further propagated to the higher layers. The next layer applies $1 \times 1$ convolution to have 3 channels which corresponds to the total number of semantic classes (i.e. road, vehicle, and background). Finally, the output feature map is fed to a soft-max classifier to obtain pixel-wise classification.

Each convolution layer in Blocks I-V (see Fig. 2) is coupled with a leaky-ReLU activation layer. We further 


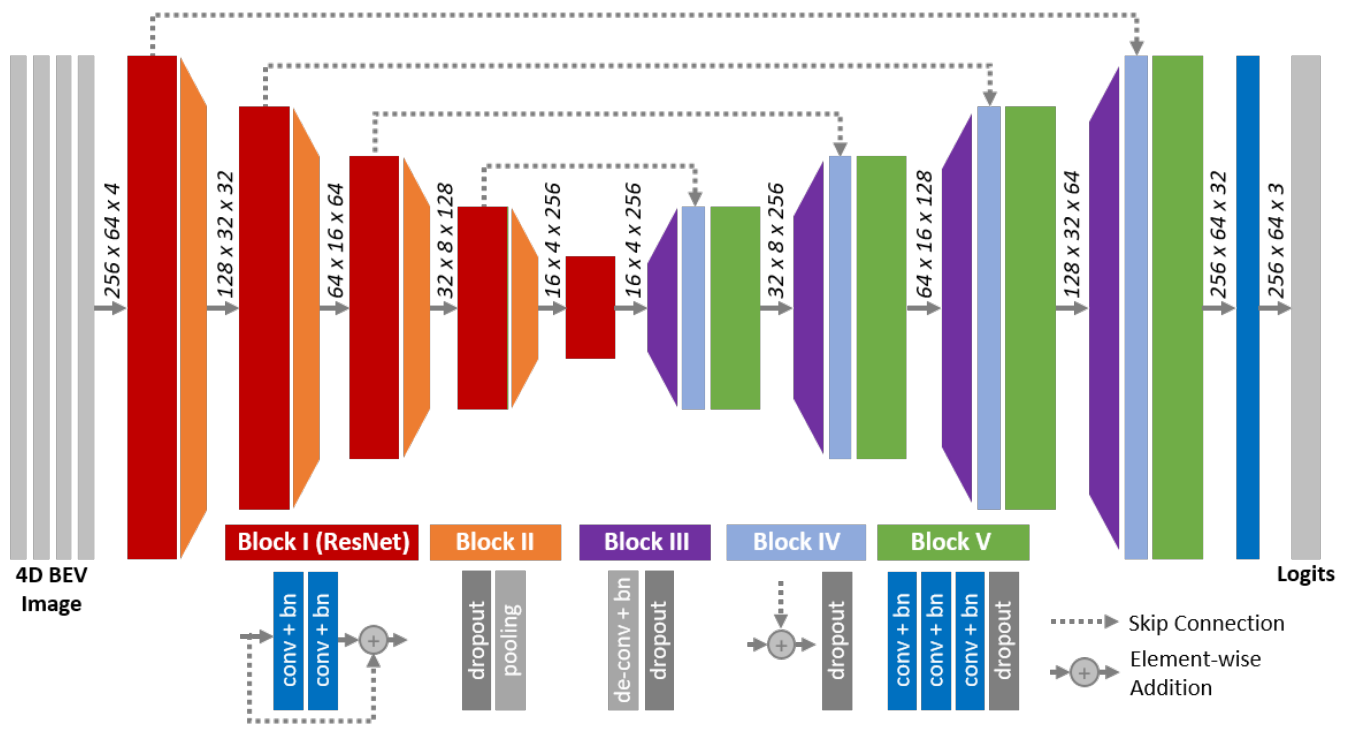

Fig. 2. Architecture of the proposed SalsaNet. Encoder part involves a series of ResNet blocks. Decoder part upsamples feature maps and combines them with the corresponding early residual block outputs using skip connections. Each convolution layer in Blocks I-V is coupled with a leaky-ReLU activation layer and a batch normalization (bn) layer.

applied batch normalization [25] after each convolution in order to help converging to the optimal solution by solving the internal covariate shift. We here emphasize that dropout needs to be placed right after batch normalization. As shown in [26], an early application of dropout can otherwise lead to a shift in the weight distribution and thus minimize the effect of batch normalization during training.

\section{Class-Balanced Loss Function}

Publicly available datasets mostly have an extreme imbalance between different classes. For instance, in the autonomous driving scenarios, vehicles appear less in the scene compared to road and background. Such an imbalance between classes yields the network to be more biased to the classes that have more samples in training and thus results in relatively poor segmentation results.

To value more the under-represented classes, we update the softmax cross-entropy loss with the smoothed frequency of each class. Our class-balanced loss function is now weighted with the inverse square root of class frequency, defined as

$$
\begin{gathered}
\mathcal{L}(y, \hat{y})=-\sum_{i} \alpha_{i} p\left(y_{i}\right) \log \left(p\left(\hat{y}_{i}\right)\right), \\
\alpha_{i}=1 / \sqrt{f_{i}},
\end{gathered}
$$

where $y_{i}$ and $\hat{y}_{i}$ are the true and predicted labels and $f_{i}$ is the frequency, i.e. the number of points, of the $i^{\text {th }}$ class. This helps the network strengthen each pixel of classes that appear less in the dataset.

\section{E. Optimizer And Regularization}

To train SalsaNet we employ the Adam optimizer [27] with the initial learning rate of 0.01 which is decayed by 0.1 after every $20 K$ iterations. The dropout probability and batch size are set to 0.5 and 32 , respectively. We run the training for 500 epochs.

To increase the amount of training data, we also augment the network input data by flipping horizontally, adding random pixel noise with probability of 0.5 , and randomly rotating about the $z$-axis in the range of $\left[-5^{\circ}, 5^{\circ}\right]$.

\section{EXPERIMENTS}

To show both the strengths and weaknesses of our model, we evaluate the performance of SalsaNet and compare with the other state-of-the-art semantic segmentation methods on the challenging KITTI dataset [9] which provides 3D LiDAR scans. We first employ the auto-labeling process described in Sec. III-A to acquire point-wise annotations. In total, we generate 10,848 point clouds where each point is labeled to one of 3 classes, i.e. road, vehicle, or background. We then follow exactly the same protocol in [6] and divide the KITTI dataset into training and test splits with 8,057 and 2,791 point clouds. We implement our model in TensorFlow and release the code and labeled point clouds for public use 1 .

\section{A. Evaluation Metric}

The performance of our model is measured on classlevel segmentation tasks by comparing each predicted point label with the corresponding ground truth annotation. As the primary evaluation metrics, we report precision $(\mathrm{P})$, recall (R), and intersection-over-union (IoU) results for each individual class as

$$
\mathrm{P}_{i}=\frac{\left|\mathcal{P}_{i} \cap \mathcal{G}_{i}\right|}{\left|\mathcal{P}_{i}\right|}, \mathrm{R}_{i}=\frac{\left|\mathcal{P}_{i} \cap \mathcal{G}_{i}\right|}{\left|\mathcal{G}_{i}\right|}, \mathrm{IoU}_{i}=\frac{\left|\mathcal{P}_{i} \cap \mathcal{G}_{i}\right|}{\left|\mathcal{P}_{i} \cup \mathcal{G}_{i}\right|},
$$

where $\mathcal{P}_{i}$ is the predicted point set of class $i$ and $\mathcal{G}_{i}$ denotes the corresponding ground truth set, whereas $|$.$| returns the$ total number of points in a set. In addition, we report the average IoU score over all the three classes.

${ }^{1}$ https://gitlab.com/aksoyeren/salsanet.git 


\begin{tabular}{|c|c|c|c|c|c|c|c|c|c|c|c|}
\hline & & \multicolumn{3}{|c|}{ Background } & \multicolumn{3}{|c|}{ Road } & \multicolumn{3}{|c|}{ Vehicle } & \multirow{2}{*}{$\begin{array}{c}\text { Average } \\
\text { IoU }\end{array}$} \\
\hline & & Precision & Recall & IoU & Precision & Recall & IoU & Precision & Recall & IoU & \\
\hline \multirow{4}{*}{ ऐ્| } & SqSeg-V1 [6] & 99.53 & 97.87 & 97.42 & 72.42 & 89.71 & 66.86 & 46.66 & 93.21 & 45.13 & 69.80 \\
\hline & SqSeg-V2 [7] & 99.39 & 98.59 & 97.99 & 77.26 & 87.33 & 69.47 & 66.28 & 90.42 & 61.93 & 76.46 \\
\hline & U-Net [14] & 99.54 & 98.47 & 98.03 & 76.08 & 90.84 & 70.65 & 67.23 & 90.54 & 62.80 & 77.27 \\
\hline & Ours & 99.46 & 98.71 & 98.19 & 78.24 & 89.39 & 71.61 & 75.13 & 89.74 & 69.19 & 79.74 \\
\hline \multirow{4}{*}{$\vec{\Delta}$} & SqSeg-V1 [6] & 97.09 & 95.02 & 92.39 & 79.72 & 83.39 & 68.79 & 70.70 & 91.50 & 66.34 & 75.84 \\
\hline & SqSeg-V2 [7] & 97.43 & 95.73 & 93.37 & 80.98 & 86.22 & 71.70 & 77.25 & 89.48 & 70.82 & 78.63 \\
\hline & U-Net [14] & 97.84 & 94.93 & 92.98 & 78.14 & 88.62 & 71.00 & 74.65 & 90.57 & 69.26 & 77.84 \\
\hline & Ours & 97.81 & 95.76 & 93.75 & 81.62 & 88.38 & 73.72 & 77.03 & 90.79 & 71.44 & 79.71 \\
\hline
\end{tabular}

TABLE I

QUANTITATIVE COMPARISON ON KITTI's TEST SET. SCORES ARE GIVEN IN PERCENTAGE (\%).

\section{B. Quantitative Results}

We compare the performance of SalsaNet with the other state-of-the-art networks: SqueezeSeg (SqSeg-V1) [6] and SqueezeSegV2 (SqSeg-V2) [7]. We particularly focus on these specialized networks because they are implemented only for the semantic segmentation task, solely rely on 3D LiDAR point clouds, and also provide open-source implementations. We train the networks SqSeg-V1 and SqSegV2 with the same configuration parameters provided in [6] and [7]. To obtain the highest score, we, however, alter the learning rate (set to 0.001 for SqSeg-V1) and also apply the same data augmentation protocol used for the training of SalsaNet. As an additional baseline method, we implement a vanilla U-Net model [14] since it is structurally similar to SalsaNet. For a fair comparison, we train U-Net with exactly the same parameters (e.g. learning rate, batch size, etc.) and strategy (e.g. loss function, data augmentation, etc.) used for the training of SalsaNet. We run our experiments for both BEV and SFV projections to study the effect of LiDAR point cloud projection on semantic segmentation. Obtained quantitative results are reported in Table I]

In all cases, our proposed model SalsaNet considerably outperforms the others by leading to the highest IoU scores. In BEV, SalsaNet particularly performs well on vehicles which are relatively small objects (e.g. compared to the road). In other methods, the highest IoU score for vehicles is $6.3 \%$ less than that of SalsaNet, which clearly indicates that these methods have difficulties extracting the local features in BEV projection. When it comes to the SFV projection, this margin between the vehicle IoU scores shrinks to $0.6 \%$ although SalsaNet still performs the best. It is because SFV has more compact form: small objects like vehicles become bigger while relatively bigger objects (such as background) occupy less portion of the SFV image (see Fig. 1). This finding indicates that SalsaNet is projection-agnostic as it can capture the local features, i.e. performs equivalently well, in both projection methods.

Furthermore, Fig. 3 depicts the final confusion matrices for SalsaNet using both BEV and SFV projections. This figure clearly presents that there is no major confusion between the classes. A small number of vehicle and road points are labeled as background but not mixed with each other. We believe that points on the road and vehicle borders in both image representations cause this minor mislabeling which can easily be overcome with more precise annotation of the training data.

\section{Qualitative Results}

For the qualitative evaluation, Fig. 4 shows some sample semantic segmentation results generated by SalsaNet using $\mathrm{BEV}$. In this figure, only for visualization purpose, segmented road and vehicle points are also projected back to the respective camera image. We, here, emphasize that these camera images have not been used for training of SalsaNet.

As depicted in Fig. 4. SalsaNet can, to a great extent, distinguish road, vehicle, and background points. All other excluded classes, e.g. cyclists on the road as shown in the first, fifth, and sixth frames in Fig. 4 are correctly segmented as background. We also illustrate a failure case in the last frame of Fig. 4 In this case, the road segment is incomplete. It is because the ground truth of the road segment only relies on the output of MultiNet [12] (see Sec. III-A) which, however, returns missing segments due to overexposure of the road from strong sunlight (see the camera image in the red frame in Fig. 4). As a potential solution, we are planning to employ a more accurate road segmentation network for camera images to increase the labeling quality of point clouds in the training dataset.

In the supplementary vided 2 , we provide more qualitative results on various KITTI scenarios.

$$
2 \text { https://youtu.be/grKnW-uGIys }
$$
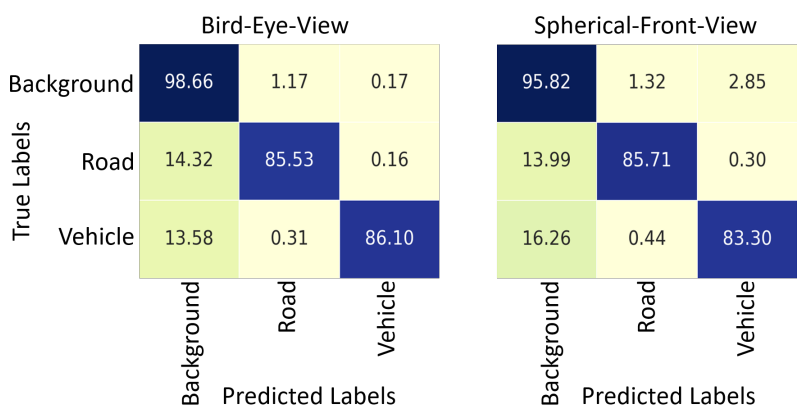

Fig. 3. The confusion matrices for our model SalsaNet using BEV and SFV projections on KITTI's test split. 

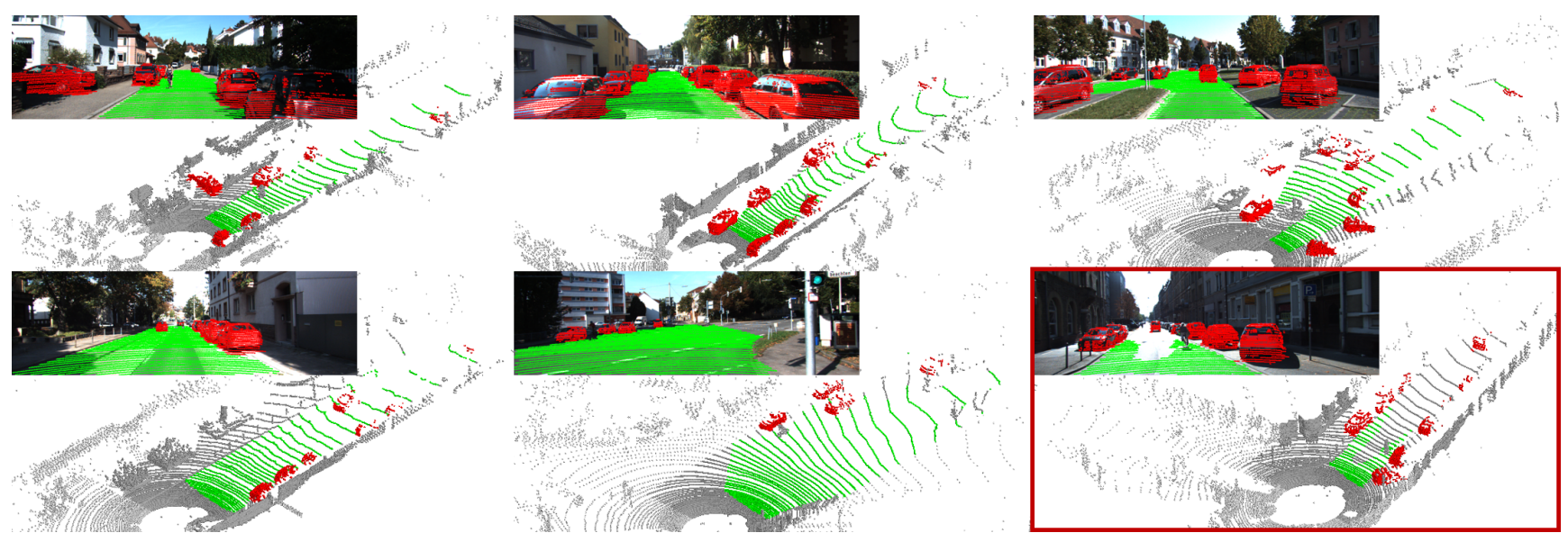

Fig. 4. Sample qualitative results showing successes and failures of our proposed method using BEV [best view in color]. Note that the corresponding camera images on the top left are only for visualization purposes and have not been used in the training. The dark- and light-gray points in the point cloud represent points that are inside and outside the bird-eye-view region, respectively. The green and red points indicate road and vehicle segments.

\section{Ablation Study}

In this ablative analysis, we investigate the individual contribution of each BEV and SFV image channel in the final performance of SalsaNet. We also diagnose the effect of using weighted loss introduced in Eg. 1 in section III-D,

Table [I] shows the obtained results for BEV. The first impression that this table conveys is that excluding weights in the loss function leads to certain accuracy drops. In particular, the vehicle IoU score decreases by $2.5 \%$, whereas the background IoU score slightly increases (see the second row in Table (II). This apparently means that the network tends to mislabel road and vehicles points since they are underrepresented in the training data. IoU scores between the third and sixth rows in the same table show that features embedded in BEV image channels have almost equal contributions to the segmentation accuracy. This clearly indicates that the BEV projection that SalsaNet employs as an input does not have any redundant information encoding.

Table III] shows the results obtained for SFV. We, again, observe the very same effect on IoU scores when the applied loss weights are omitted. The most interesting findings, however, emerge when we start measuring the contribution of SFV image channels. As the last six rows in Table III indicate, SFV image channels have rather inconsistent effects

\begin{tabular}{|c|c|c|c|c|c|c|c|c|c|}
\hline & \multicolumn{4}{|c|}{ Channels } & \multirow{2}{*}{$\begin{array}{c}\text { Loss } \\
\text { Weight }\end{array}$} & \multicolumn{4}{|c|}{ IoU } \\
\hline & Mean & Max & Ref & Dens & & Background & Road & Vehicle & Average \\
\hline \multirow{6}{*}{ 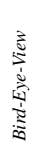 } & $\checkmark$ & $\checkmark$ & $\checkmark$ & $\checkmark$ & $\checkmark$ & 98.19 & 71.61 & 69.19 & 79.74 \\
\hline & $\checkmark$ & $\checkmark$ & $\checkmark$ & $\checkmark$ & - & 98.23 & 71.52 & 66.70 & 78.91 \\
\hline & - & $\checkmark$ & $\checkmark$ & $\checkmark$ & $\checkmark$ & 98.13 & 71.30 & 66.70 & 78.81 \\
\hline & $\checkmark$ & - & $\checkmark$ & $\checkmark$ & $\checkmark$ & 98.20 & 71.46 & 67.98 & 79.33 \\
\hline & $\checkmark$ & $\checkmark$ & - & $\checkmark$ & $\checkmark$ & 98.13 & 71.02 & 62.46 & 77.30 \\
\hline & $\checkmark$ & $\checkmark$ & $\checkmark$ & - & $\checkmark$ & 98.13 & 71.04 & 67.42 & 78.95 \\
\hline
\end{tabular}

TABLE II

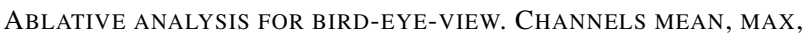
REF, AND DENS STAND FOR THE MEAN AND MAXIMUM ELEVATION, REFLECTIVITY, AND NUMBER OF PROJECTED POINTS IN ORDER. on the segmentation accuracy. For instance, while adding the mask channel increases the average accuracy by $4.5 \%$, the first two channels that keep $\mathrm{x}-\mathrm{y}$ coordinates lead to drop in overall average accuracy by $0.04 \%$ and $0.34 \%$, respectively. This is a clear evidence that the SFV projection introduced in [6] and [7] contains redundant information that mislead the feature learning in networks.

Given these findings and also the arguments regarding the deformation in SFV as stated in section III-B.2, we conclude that the BEV projection is more appropriate point cloud representation. Thus, SalsaNet relies on BEV.

\section{E. Runtime Evaluation}

Runtime performance is of utmost importance in autonomous driving. Table IV]reports the forward pass runtime performance of SalsaNet in contrast to other networks. To obtain fair statistics, all measurements are repeated 10 times using all the test data on the same NVIDIA Tesla V100DGXS-32GB GPU card. Obtained mean runtime values together with standard deviations are presented in Table IV Our method clearly exhibits better performance compared to SqSeg-V1 and SqSeg-V2 in both projection methods, BEV and SFV. We observed that U-Net performs slightly better than SalsaNet. There is, however, a trade-off since U-Net

\begin{tabular}{|c|c|c|c|c|c|c|c|c|c|c|c|}
\hline & \multicolumn{6}{|c|}{ Channels } & \multirow{2}{*}{$\begin{array}{c}\text { Loss } \\
\text { Weight }\end{array}$} & \multicolumn{4}{|c|}{ IoU } \\
\hline & $\mathrm{X}$ & $\mathrm{Y}$ & Z & I & $\mathrm{R}$ & M & & Background & Road & Vehicle & Average \\
\hline \multirow{8}{*}{ 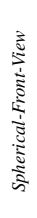 } & $\checkmark$ & $\checkmark$ & $\checkmark$ & $\checkmark$ & $\checkmark$ & $\checkmark$ & $\checkmark$ & 93.75 & 73.72 & 71.44 & 79.71 \\
\hline & $\checkmark$ & $\checkmark$ & $\checkmark$ & $\checkmark$ & $\checkmark$ & $\checkmark$ & - & 93.90 & 73.80 & 70.30 & 79.43 \\
\hline & - & $\checkmark$ & $\checkmark$ & $\checkmark$ & $\checkmark$ & $\checkmark$ & $\checkmark$ & 93.76 & 73.97 & 71.34 & 79.75 \\
\hline & $\checkmark$ & - & $\checkmark$ & $\checkmark$ & $\checkmark$ & $\checkmark$ & $\checkmark$ & 94.00 & 74.61 & 71.21 & 80.05 \\
\hline & $\checkmark$ & $\checkmark$ & - & $\checkmark$ & $\checkmark$ & $\checkmark$ & $\checkmark$ & 93.90 & 74.72 & 69.77 & 79.50 \\
\hline & $\checkmark$ & $\checkmark$ & $\checkmark$ & - & $\checkmark$ & $\checkmark$ & $\checkmark$ & 93.07 & 72.87 & 66.06 & 77.41 \\
\hline & $\checkmark$ & $\checkmark$ & $\checkmark$ & $\checkmark$ & - & $\checkmark$ & $\checkmark$ & 93.19 & 73.73 & 64.57 & 77.30 \\
\hline & $\checkmark$ & $\checkmark$ & $\checkmark$ & $\checkmark$ & $\checkmark$ & - & $\checkmark$ & 92.46 & 73.29 & 59.53 & 75.20 \\
\hline
\end{tabular}

TABLE III

Ablative anALYSiS For SPHERICAL-FRONT-VIEW. ChanNels $\mathrm{X}, \mathrm{Y}, \mathrm{Z}$,

$\mathrm{I}, \mathrm{R}$, AND M STAND FOR THE CARTESIAN COORDINATES $(x, y, z)$, INTENSITY, RANGE, AND OCCUPANCY MASK, IN ORDER. 


\begin{tabular}{rlccc}
\hline & & Mean (msec) & Std (msec) & Speed (fps) \\
\hline & SqSeg-V1 [6] & 6.77 & 0.31 & $148 \mathrm{~Hz}$ \\
SqSeg-V2 [7] & 10.24 & 0.31 & $98 \mathrm{~Hz}$ \\
U. & $\mathbf{5 . 3 1}$ & 0.21 & $\mathbf{1 8 8 ~ H z}$ \\
& U-Net [14] & 6.26 & 0.08 & $160 \mathrm{~Hz}$ \\
\hline Ours & 6.92 & 0.21 & $144 \mathrm{~Hz}$ \\
& SqSeg-V1 [6] & 10.36 & 0.41 & $96 \mathrm{~Hz}$ \\
SqSeg-V2 [7] & $\mathbf{5 . 4 5}$ & 0.21 & $\mathbf{1 8 3} \mathrm{Hz}$ \\
U-Net [14] & 6.41 & 0.13 & $156 \mathrm{~Hz}$ \\
\hline
\end{tabular}

TABLE IV

RUNTIME PERFORMANCE ON KITTI DATASET [9]

returns relatively lower accuracies (see Table I). The reason why U-Net is performing faster is because of the relatively less number of kernels used in each network layer. We here note that the standard deviation of the SalsaNet runtime is much less than the others. This plays a vital role in the stability of the self-driving perception modules. Lastly, all the methods are getting faster in case of using BEV. This can be explained by the fact that the channel number and resolution in BEV images are slightly less.

Overall, we conclude that our network inference (a single forward pass) time can reach up to $160 \mathrm{~Hz}$ while providing the highest accuracies in BEV. Note that this high speed is significantly faster than the sampling rate of mainstream LiDAR scanners which typically work at $10 \mathrm{~Hz}$ [9].

\section{CONCLUSION}

In this work, we presented a new deep network SalsaNet to semantically segment road, i.e. drivable free-space, and vehicle points in real-time using 3D LiDAR data only. Our method differs in that SalsaNet is input-data agnostic, that means performs equivalently well in both BEV and SFV projections although other well-known semantic segmentation networks [6], [7], [14] have difficulties extracting the local features in the BEV projection. By directly transferring image-based point-wise semantic information to the point cloud, our proposed method can automatically generate a large set of annotated LiDAR data required for training.

Consequently, SalsaNet is simple, fast, and returns stateof-the-art results. Our extensive quantitative and qualitative experimental evaluations present intuitive understanding of the strengths and weaknesses of SalsaNet compared to alternative methods. Application of SalsaNet to bootstrap the detection and tracking processes is our planned future task in the context of autonomous driving.

\section{REFERENCES}

[1] R. P. K. Poudel, S. Liwicki, and R. Cipolla, "Fast-scnn: Fast semantic segmentation network," CoRR, vol. abs/1902.04502, 2019. [Online]. Available: http://arxiv.org/abs/1902.04502

[2] P. G. Meyer, J. Charland, D. Hegde, A. Laddha, and C. VallespiGonzalez, "Sensor fusion for joint 3d object detection and semantic segmentation," in https://arxiv.org/pdf/1904.11466.pdf, 2019.

[3] C. R. Qi, W. Liu, C. Wu, H. Su, and L. J. Guibas, "Frustum pointnets for 3d object detection from RGB-D data," CoRR, 2017. [Online]. Available: http://arxiv.org/abs/1711.08488
[4] L. Caltagirone, S. Scheidegger, L. Svensson, and M. Wahde, "Fast lidar-based road detection using fully convolutional neural networks," in IEEE Intelligent Vehicles Symposium, 2017, pp. 1019-1024.

[5] M. Velas, M. Spanel, M. Hradis, and A. Herout, "Cnn for very fast ground segmentation in velodyne lidar data," in IEEE Int. Conf. on Autonomous Robot Systems and Competitions, 2018, pp. 97-103.

[6] B. Wu, A. Wan, X. Yue, and K. Keutzer, "Squeezeseg: Convolutional neural nets with recurrent crf for real-time road-object segmentation from 3d lidar point cloud," ICRA, 2018.

[7] B. Wu, X. Zhou, S. Zhao, X. Yue, and K. Keutzer, "Squeezesegv2: Improved model structure and unsupervised domain adaptation for road-object segmentation from a lidar point cloud," in ICRA, 2019.

[8] K. He, X. Zhang, S. Ren, and J. Sun, "Deep residual learning for image recognition," in Proceedings of the IEEE conference on computer vision and pattern recognition, 2016, pp. 770-778.

[9] A. Geiger, P. Lenz, and R. Urtasun, "Are we ready for autonomous driving? the kitti vision benchmark suite," in Conference on Computer Vision and Pattern Recognition (CVPR), 2012.

[10] F. Piewak, P. Pinggera, M. Schäfer, D. Peter, B. Schwarz, N. Schneider, D. Pfeiffer, M. Enzweiler, and J. M. Zöllner, "Boosting lidar-based semantic labeling by cross-modal training data generation," CoRR, vol. abs/1804.09915, 2018.

[11] B. Wang, V. Wu, B. Wu, and K. Keutzer, "LATTE: accelerating lidar point cloud annotation via sensor fusion, one-click annotation, and tracking," CoRR, vol. abs/1904.09085, 2019.

[12] M. Teichmann, M. Weber, M. Zllner, R. Cipolla, and R. Urtasun, "Multinet: Real-time joint semantic reasoning for autonomous driving," in IEEE Intelligent Vehicles Symposium, 2018, pp. 1013-1020.

[13] K. He, G. Gkioxari, P. Dollár, and R. B. Girshick, "Mask R-CNN," CoRR, vol. abs/1703.06870, 2017. [Online]. Available: http://arxiv.org/abs/1703.06870

[14] O. Ronneberger, P.Fischer, and T. Brox, "U-net: Convolutional networks for biomedical image segmentation," in Medical Image Computing and Computer-Assisted Intervention (MICCAI), ser. LNCS, vol. 9351. Springer, 2015, pp. 234-241.

[15] D. Zermas, I. Izzat, and N. Papanikolopoulos, "Fast segmentation of $3 \mathrm{~d}$ point clouds: A paradigm on lidar data for autonomous vehicle applications," in 2017 IEEE International Conference on Robotics and Automation (ICRA), May 2017, pp. 5067-5073.

[16] L. Chen, J. Yang, and H. Kong, "Lidar-histogram for fast road and obstacle detection," in 2017 IEEE International Conference on Robotics and Automation (ICRA), May 2017, pp. 1343-1348.

[17] Y. Wang, T. Shi, P. Yun, L. Tai, and M. Liu, "Pointseg: Real-time semantic segmentation based on 3d lidar point cloud," CoRR, vol. abs/1807.06288, 2018.

[18] B. Yang, W. Luo, and R. Urtasun, "PIXOR: real-time 3d object detection from point clouds," CoRR, vol. abs/1902.06326, 2019. [Online]. Available: http://arxiv.org/abs/1902.06326

[19] E. Shelhamer, J. Long, and T. Darrell, "Fully convolutional networks for semantic segmentation." PAMI, 2016.

[20] C. Zhang, W. Luo, and R. Urtasun, "Efficient convolutions for realtime semantic segmentation of $3 \mathrm{~d}$ point clouds," in Proceedings of the International Conference on 3D Vision (3DV), 2018.

[21] Y. Zhou and O. Tuzel, "Voxelnet: End-to-end learning for point cloud based 3d object detection," in 2018 IEEE/CVF Conference on Computer Vision and Pattern Recognition, June 2018, pp. 4490-4499.

[22] C. R. Qi, H. Su, K. Mo, and L. J. Guibas, "Pointnet: Deep learning on point sets for 3d classification and segmentation," CoRR, 2016. [Online]. Available: http://arxiv.org/abs/1612.00593

[23] Y. Zeng, Y. Hu, S. Liu, J. Ye, Y. Han, X. Li, and N. Sun, "Rt3d: Real-time 3-d vehicle detection in lidar point cloud for autonomous driving," IEEE Robotics and Automation Letters, vol. 3, no. 4, pp. 3434-3440, Oct 2018.

[24] M. Simon, S. Milz, K. Amende, and H. Gross, "Complex-yolo: Realtime 3d object detection on point clouds," CoRR, 2018.

[25] S. Ioffe and C. Szegedy, "Batch normalization: Accelerating deep network training by reducing internal covariate shift," arXiv preprint arXiv:1502.03167. 2015.

[26] X. Li, S. Chen, X. Hu, and J. Yang, "Understanding the disharmony between dropout and batch normalization by variance shift," arXiv preprint arXiv:1801.05134 2018.

[27] D. P. Kingma and J. Ba, "Adam: A method for stochastic optimization," 2014. 